\title{
Neugebauer and Demichel: Dependence and Independence in $n$-Screen Superpositions for Colour Printing
}

\section{Isaac Amidror, Roger D. Hersch}

\author{
Laboratoire de Systèmes Périphériques, Ecole Polytechnique Fédérale de Lausanne, Lausanne, Switzerland
}

Received 3 February 1999; accepted 20 September 1999

\begin{abstract}
The Neugebauer equations and the Demichel equations on which they are based are one of the basic tools for modeling colour printing systems that use the halftoning technique. However, these equations implicitly assume that the colour ink distributions in the screen superposition are statistically independent. We show that this condition is not satisfied in the conventional screen superposition used for colour printing, and we discuss the consequences of this fact. Furthermore, we give a precise criterion that determines, for any number of superposed regular screens, in which cases the Demichel (and hence the Neugebauer) equations are satisfied, and in which cases they fail: The Demichel equations fail in all cases where the screen superposition is singular, and they are satisfied in all nonsingular screen superpositions. We illustrate our results with several examples of both cases. $\odot 2000$ John Wiley \& Sons, Inc. Col Res Appl, 25, 267-277, 2000
\end{abstract}

Key words: Neugebauer equations; Demichel equations; colour printing; dot-screen superpositions

\section{INTRODUCTION}

The Neugebauer equations are one of the most basic tools for modeling colour printing systems that are based on halftoning techniques. ${ }^{1}$ The use of the Neugebauer equations for predicting the colour of a given printed patch requires a precise knowledge of the proportional area of each ink combination within the given patch. This information can be obtained by counting pixels (either under a microscope or by using a computer simulation), but most

\footnotetext{
Correspondence to: Dr. Isaac Amidror, Laboratoire de Systèmes Périphériques, Ecole Polytechnique Fédérale de Lausanne, CH-1015 Lausanne, Switzerland

(C) 2000 John Wiley \& Sons, Inc.
}

often this is done by direct calculation, using the Demichel equations. However, the Demichel equations are valid only under a rather strict condition: they require that the superposed screens be independent of each other, so that the percent area of dot overlap in the superposition be the product of the individual dot percent areas in the original halftone screens.

It has been recently shown, ${ }^{2}$ as a consequence of this requirement, that in the superposition of two regular dotscreens having the same frequency* the validity of the Demichel equations is not always guaranteed, and it depends on the angle between the two superposed screens.

In the present contribution, we extend this result to the most general case of regular screen superpositions, namely the superposition of $n$ screens with any angle and frequency combinations. We show that the Demichel equations are valid only for screen superpositions that are nonsingular, while for singular superpositions, ${ }^{\dagger}$ including the conventional 3- or 4-screen superpositions that are traditionally used in colour printing, the Demichel equations are not valid. Although the colour deviations that result from using the Demichel equations in such singular cases are not excessively large, they still may be nonnegligible, and one should be aware of their existence when using the Neugebauer equations.

\section{THE NEUGEBAUER AND DEMICHEL EQUATIONS}

Most printing devices are only bilevel, meaning that they are only capable of printing solid ink or leaving the paper unprinted, but they cannot produce intermediate ink tones.

\footnotetext{
* The frequency of a regular dot-screen is defined as the number of screen dots per unit of length (inch, $\mathrm{cm}$, etc.) along the screen's main axes.

$\dagger$ The precise mathematical meaning of these terms is explained below, following Proposition 1.
} 
TABLE I. The $2^{3}=8$ possible combinations of ink superposition in the classical case with cyan, magenta, and yellow inks.

\begin{tabular}{cccc}
\multicolumn{2}{c}{ Primary inks $\mathrm{C}_{1}, \mathrm{C}_{2}, \mathrm{C}_{3}:$} & \multicolumn{1}{c}{$\begin{array}{c}\text { Colour resulting } \\
\text { from the ink superposition }\end{array}$} \\
\cline { 1 - 2 } $\mathrm{C}$ & $\mathrm{M}$ & $\mathrm{Y}$ & $\mathrm{W}$ (white) \\
0 & 0 & 0 & $\mathrm{C}$ (cyan) \\
1 & 0 & 0 & $\mathrm{M}$ (magenta) \\
0 & 1 & 0 & $\mathrm{Y}$ (yellow) \\
0 & 0 & 1 & $\mathrm{R}$ (red) \\
0 & 1 & 1 & $\mathrm{G}$ (green) \\
1 & 0 & 1 & B (blue) \\
1 & 1 & 0 & $\mathrm{~K}$ (black) \\
1 & 1 & 1 &
\end{tabular}

1 and 0 indicate, respectively, if the ink in question does or does not participate in the superposition.

In such devices the visual impression of intermediate tone levels is usually obtained by means of the halftoning technique, i.e., by breaking the original continuous-tone image into small dots whose size varies depending on the tone level. This is also true for most colour printing devices, where each of the primary inks or colorants (usually: cyan $(\mathrm{C})$, magenta $(\mathrm{M})$, yellow $(\mathrm{Y})$ and often also black $(\mathrm{K})$ ) is only bilevel. The principle of colour image printing is based on the fact that a wide range of colours can be obtained, or at least approximated, by combinations of these primary colour inks. The original colour image is first separated, either by photographic or by digital filtering, into 3 or 4 layers (colour planes), one for each of the CMY or CMYK primary ink colours. Then, the halftoning technique is applied separately to each of the colour planes of the image, and the resulting 3 or 4 colour screens are printed one on top of the other. This gives to the eye, when looking from a sufficient distance, an illusion of a full range of intermediate colour levels, although the printing device is only bilevel.

The Neugebauer equations ${ }^{1}$ are the basic mathematical tool for modeling such bilevel colour printing systems that use the halftoning technique. The Neugebauer equations are based on the fact that printing $n$ superposed dot-screens using the colour inks $\mathrm{C}_{1}, \ldots, \mathrm{C}_{n}$ gives rise to $2^{n}$ possible combinations of ink superpositions on each point of the printed paper. Table I shows the $2^{3}=8$ possible ink overlaps for the classical case with $n=3$ inks: cyan, magenta, and yellow. For the sake of simplicity, in the following discussion we mainly use the case with $n=3$ inks, but the generalization to any number $n$ is straightforward.

In order to establish the Neugebauer equations, we need to know first the tristimulus $X Y Z$ colour coordinates of the inks to be used and of all their possible superpositions. In our 3-colour example we denote these coordinates by $X_{i j k}, Y_{i j k}$, and $Z_{i j k}$, where the binary indices $i, j, k$ run over the $2^{3}$ colour combinations as shown in Table I. These colorimetric coordinates can be obtained by printing a sample patch for each of the $2^{3}$ possible superpositions of solid (i.e., $100 \%$ coverage) inks, and measuring their $X Y Z$ values using a colorimeter.

Suppose now that we are given a new halftoned patch that has been printed using the same CMY inks. Depending on the case, this patch may consist of one, two, or three superposed dot-screens, each screen being printed with one of the C, M, Y inks. Looking through a microscope we can see that our printed patch is covered by a multitude of partially overlapping cyan, magenta, and yellow halftone dots, possibly separated by some unprinted areas. Now, if we know the fraction of the total patch area (i.e., the relative area) $a_{i j k}$ that is occupied by each of the possible $2^{3}$ ink overlaps in our patch, then, by virtue of the additive colour composition principle, ${ }^{3}$ we can predict the $X Y Z$ colour coordinates of our patch by means of the following equations, known as the Neugebauer equations:

$$
\begin{aligned}
& X_{P}=a_{000} X_{000}+a_{001} X_{001}+\ldots+a_{111} X_{111}, \\
& Y_{P}=a_{000} Y_{000}+a_{001} Y_{001}+\ldots+a_{111} Y_{111}, \\
& Z_{P}=a_{000} Z_{000}+a_{001} Z_{001}+\ldots+a_{111} Z_{111} .
\end{aligned}
$$

The surface fractions $a_{i j k}$ can be found, for example, by counting inside our given patch the number of pixels covered by each of the $2^{3}$ possible ink combinations, taking into account the exact halftone dot shapes and sizes. However, as we see below, under certain conditions it is possible to calculate these coefficients in a much simpler way.

Let us denote by $P(\mathrm{C})=c, P(\mathrm{M})=m$, and $P(\mathrm{Y})=y$ the fractions of the patch area, where the $\mathrm{C}, \mathrm{M}$, and $\mathrm{Y}$ inks have been deposited. From a statistical point of view, $P(\mathrm{C})$, $P(\mathrm{M})$, and $P(\mathrm{Y})$ may also be considered as the probability of any particular point that is randomly chosen within the patch area being covered by the $\mathrm{C}, \mathrm{M}$, or $\mathrm{Y}$ ink, respectively. It follows that the probability of not being covered by cyan is $P(\neg \mathrm{C})=1-c$ (where $\neg$ is the NOT symbol), that of not being covered by magenta is $P(\neg \mathrm{M})=1-m$, and that of not being covered by yellow is $P(\neg \mathrm{Y})=1-y$. Hence, the probability of any randomly chosen point being covered by all three colours is $P(\mathrm{C}) P(\mathrm{M}) P(\mathrm{Y})=c m y$, and the probability of not being covered by any of the three colours is $P(\neg \mathrm{C}) P(\neg \mathrm{M}) P(\neg \mathrm{Y})=(1-c)(1-m)(1-y)$. Similarly, the probability of any point of only being covered by cyan is $P(\mathrm{C}) P(\neg \mathrm{M}) P(\neg \mathrm{Y})=c(1-m)(1-y)$ (note that this is not the same as the probability of being covered by cyan, $P(\mathrm{C})$ !). By similar reasoning, we can predict the surface fraction $a_{i j k}$ covered by each of the $2^{3}$ ink combinations of Table I as a function of the dot percentages $c, m$, and $y$ of the individual $\mathrm{C}-, \mathrm{M}-$, and $\mathrm{Y}$-screens in our patch:

$$
\begin{aligned}
& a_{000}=P(\neg \mathrm{C}) P(\neg \mathrm{M}) P(\neg \mathrm{Y})=(1-c)(1-m)(1-y) \\
& a_{100}=P(\mathrm{C}) P(\neg \mathrm{M}) P(\neg \mathrm{Y})=c(1-m)(1-y) \\
& a_{010}=P(\neg \mathrm{C}) P(\mathrm{M}) P(\neg \mathrm{Y})=(1-c) m(1-y) \\
& a_{001}=P(\neg \mathrm{C}) P(\neg \mathrm{M}) P(\mathrm{Y})=(1-c)(1-m) y \\
& a_{011}=P(\neg \mathrm{C}) P(\mathrm{M}) P(\mathrm{Y})=(1-c) m y \\
& a_{101}=P(\mathrm{C}) P(\neg \mathrm{M}) P(\mathrm{Y})=c(1-m) y \\
& a_{110}=P(\mathrm{C}) P(\mathrm{M}) P(\neg \mathrm{Y})=c m(1-y) \\
& a_{111}=P(\mathrm{C}) P(\mathrm{M}) P(\mathrm{Y})=c m y .
\end{aligned}
$$


These equations, known as the Demichel equations, were first published in 1924 in a now forgotten French printer's review called "Le Procédé."4 By substituting these equations in the Neugebauer Eqs. (1), we obtain a prediction of the $X Y Z$ values of the printed colour patch in question.

Note, however, that in the above reasoning it has been tacitly assumed that the distributions of the cyan, magenta, and yellow inks in the screen superposition are independent; we return to this point in more detail in a later section, and once again in Appendix A.

\section{MACRO- AND MICROSTRUCTURES IN THE SUPERPOSITION OF REGULAR SCREENS}

Although the considerations leading to the Demichel equations seem to be quite plausible, in reality they are not valid in all cases. It has been shown recently, ${ }^{2}$ for the case of 2-screen superpositions, that the Demichel considerations are not generally true for all screen superposition angles. Still worse: as we see below, it turns out that even in the very case for which they were originally devised, namely the conventional superposition of 3 regular screens with identical frequencies and angle differences of $30^{\circ}$ (or $60^{\circ}$ ), the Demichel equations do not really hold. In order to demonstrate the problem, we need to make a small digression on the microstructure of dot-screen superpositions (a more detailed discussion on this subject can be found in Chapter 8 of Ref. 5).

It is well known that when periodic layers (such as line grids, dot-screens, etc.) are superposed, new structures of two distinct levels may appear in the superposition, which do not exist in any of the original layers: the macrostructures and the microstructures. The macrostructures, usually known as the moiré patterns, are, of course, the most prominent; being much coarser than the detail of the original layers, they are clearly visible even when observed from a distance. The microstructures, on the contrary, are almost as small as the periods of the original screens (typically, just 2-5 times larger), and, therefore, they are visible only when examining the superposition from a close distance or through a magnifying glass. These tiny structures are also called rosettes due to the various flower-like shapes they often form in the superposition of dot-screens (Ref. 6, p. 339).

Macrostructures and microstructures may coexist in the same superposition; in fact, macrostructures, whenever they exist, are made up from repetitive alternations in the microstructures. However, while microstructures exist practically in any screen superposition, except for the most trivial cases, macro moiré effects are not always generated. A short discussion on the relationship between macro- and microstructures is given in Appendix B; a more complete account can be found in Chapter 8 of Ref. 5. In the present article we are not concerned with macrostructures, since we are only interested here in moiré-free screen superpositions that are usable in colour printing. Therefore, we concentrate now on the microstructures (the rosettes) that are generated in screen superpositions.
Most famous are the rosette forms that are obtained in the classical superposition of three identical screens with equal angle differences of $30^{\circ}$ ( or $60^{\circ}$ ); these rosette forms are well known in the printing industry and they have been widely described in literature (Ref. 6, pp. 339-341; Ref. 7, pp. 57-59; Ref. 8). If we examine the forms of these rosettes in various registration positions of the superposed screens, i.e., when the original screens are being laterally shifted with respect to each other, we can distinguish between two main types of rosettes. When the three screens are superposed in-phase, i.e., with a dot centered on the origin, a perfect match of one screen-dot from each layer occurs at the origin, generating there a "dot-centered" rosette. Moreover, almost perfect copies of this dot-centered rosette appear all over the superposition, generating a uniform microstructure with almost-dot-centered rosettes throughout. This is illustrated in Fig. 1(c) and in its magnified version in Fig. 2(c). However, when the screens are superposed in counterphase, a different pattern of "clear-centered" rosettes is generated throughout the superposition [see Figs. 1(d) and 2(d)]. A gradual transition between these extreme rosetteforms occurs in the intermediate phase positions. Note, however, that each type of rosette manifests itself in a different shift combination of the original screens, so that different rosette types do not mix together in the same superposition.

However, the microstructure obtained in a superposition of only two of the three screens with the same angle difference of $30^{\circ}$ (or $60^{\circ}$ ) is characterized by the simultaneous presence of microstructure elements of all types (dot-centered, clear-centered, and intermediate variants), which are uniformly distributed throughout the superposition plane. This gives to the eye the impression of a uniform and regular microstructure, as shown in Fig. 1(a) and in its magnified version in Fig. 2(a). Furthermore, in this 2-screen superposition, in contrast to its 3 -screen counterpart, registration shifts between the individual screens do not modify the general nature of the microstructure [compare Figs. 1(a) and 1(b)].

\section{LIMITATIONS OF THE DEMICHEL CONSIDERATIONS}

This difference in the behavior of the microstructure between the classical 3-screen superposition and its 2-screen counterpart has an important consequence on the colorimetric behavior of these screen superpositions.

Suppose that the 3-screen superposition consists of a C-screen, an M-screen and a Y-screen, each of which having a constant dot size throughout. It is clear, therefore, that in the C-screen $P(\mathrm{C})$ and $P(\neg \mathrm{C})=1-P(\mathrm{C})$ are constant and invariant under any possible shifts of the dot-screen. A similar reasoning is also true for $P(\mathrm{M})$ and $P(\neg \mathrm{M})=1-$ $P(\mathrm{M})$ in the $\mathrm{M}$-screen, and for $P(\mathrm{Y})$ and $P(\neg \mathrm{Y})=1-P(\mathrm{Y})$ in the Y-screen.

Consequently, it follows that both $P(\mathrm{C}) P(\mathrm{M}) P(\mathrm{Y})$ and $P(\neg \mathrm{C}) P(\neg \mathrm{M}) P(\neg \mathrm{Y})$ are constant, and they are not influenced by possible shifts in any of the dot-screens.

However, as it is well known in the printing art (Ref. 6, 

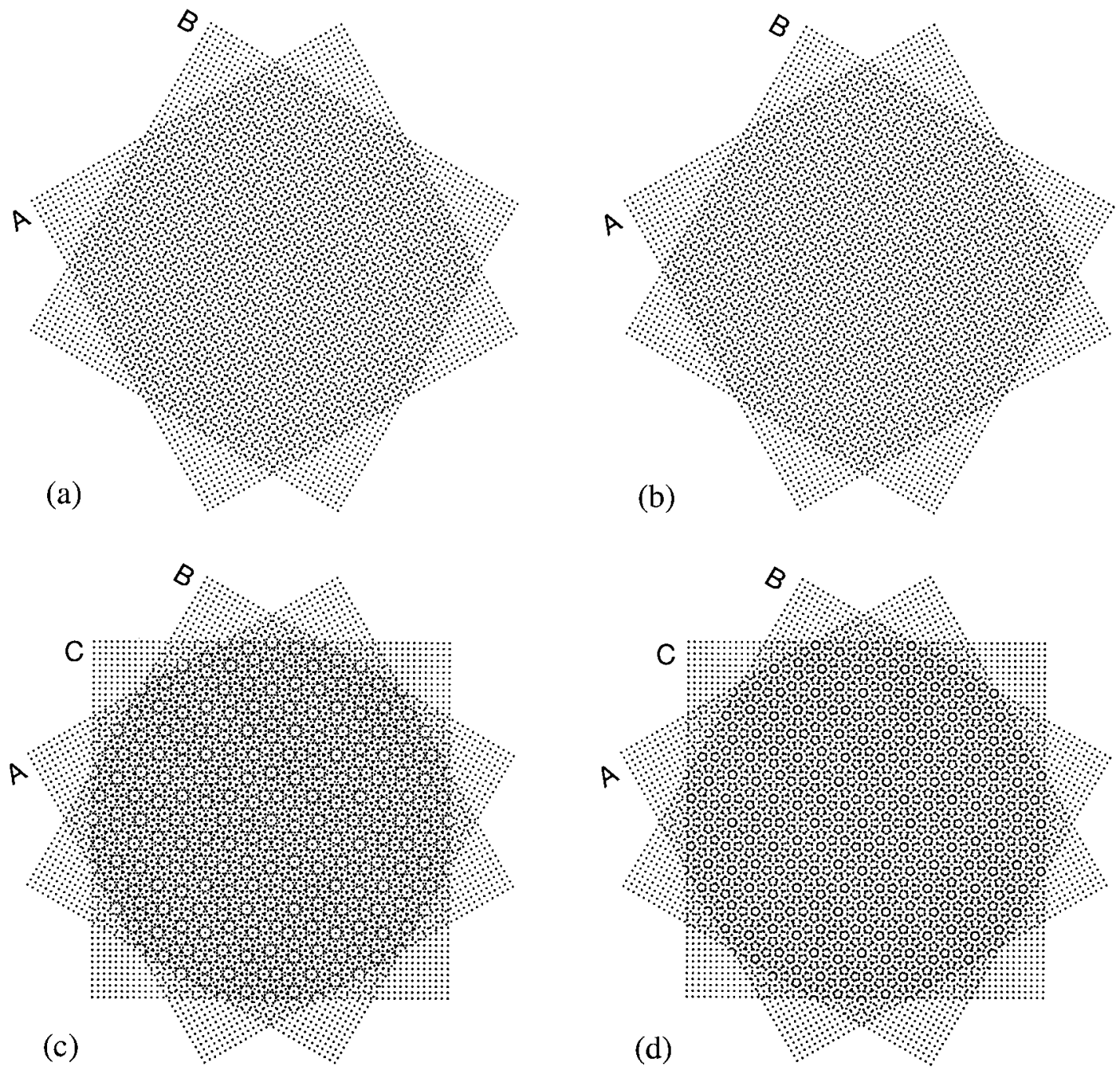

FIG. 1. (a) In-phase superposition of two identical dot-screens at angles $\theta_{1}=30^{\circ}$ and $\theta_{2}=-30^{\circ}$. (b) Counter-phase superposition of the same screens. (c) In-phase superposition with a third identical screen having angle $\theta_{3}=0^{\circ}$ on top of (a). (d) Counter-phase superposition with a third identical screen having angle $\theta_{3}=0^{\circ}$ on top of (b).

pp. 339, 341-342), it turns out that in the classical 3-screen superposition there exists between dot-centered and clearcentered rosettes slight differences in the fractions of ink dot coverage. For example, as we can see from Figs. 2(c),(d) and from Table II, in the in-phase superposition, where the microstructure consists of dot-centered rosettes, there is slightly more area that is simultaneously covered by the 3 colorants than in the counter-phase superposition, where the microstructure consists of clear-centered rosettes and no dot-centered rosettes are found. This means that $P(\mathrm{~K})=$ $P\left(\mathrm{C}^{\wedge} \mathrm{M}^{\wedge} \mathrm{Y}\right)$ (where ${ }^{\wedge}$ is the AND symbol) does vary according to the shift registration of the individual dot-screens in the superposition. Consequently, although there may exist a particular shift combination in which $P\left(\mathrm{C}^{\wedge} \mathrm{M}^{\wedge} \mathrm{Y}\right)=$ $P(\mathrm{C}) P(\mathrm{M}) P(\mathrm{Y})$, this equality does not hold in all possible shifts. In general, we have, therefore:

$$
P\left(\mathrm{C}^{\wedge} \mathrm{M}^{\wedge} \mathrm{Y}\right) \neq P(\mathrm{C}) P(\mathrm{M}) P(\mathrm{Y}) .
$$

This means that in the classical 3-screen superposition the three superposed screens are not independent of each other.
TABLE II. The proportional areas of each of the $2^{3}=$ 8 possible combinations of ink superposition in the classical 3-screen superposition, which is a singular case, in in-phase and in counter-phase superpositions.

\begin{tabular}{|c|c|c|c|}
\hline Ink overlap & $\begin{array}{l}\text { Proportional } \\
\text { area in } \\
\text { in-phase } \\
\text { superposition }\end{array}$ & $\begin{array}{l}\text { Proportional } \\
\text { area in } \\
\text { counter-phase } \\
\text { superposition }\end{array}$ & Difference \\
\hline $\mathrm{W}=\neg \mathrm{C}^{\wedge} \neg \mathrm{M}^{\wedge} \neg \mathrm{Y}$ & 0.2051 & 0.2390 & -0.0339 \\
\hline$C=C^{\wedge} \neg M^{\wedge} \neg Y$ & 0.1754 & 0.1410 & 0.0344 \\
\hline $\mathrm{M}=\neg \mathrm{C}^{\wedge} \mathrm{M}^{\wedge} \neg \mathrm{Y}$ & 0.1739 & 0.1394 & 0.0345 \\
\hline $\mathrm{Y}=\neg \mathrm{C}^{\wedge} \neg \mathrm{M}^{\wedge} \mathrm{Y}$ & 0.1740 & 0.1399 & 0.0341 \\
\hline$R=\neg C^{\wedge} M^{\wedge} Y$ & 0.0620 & 0.0967 & -0.0347 \\
\hline $\mathrm{G}=\mathrm{C}^{\wedge} \neg \mathrm{M}^{\wedge} \mathrm{Y}$ & 0.0612 & 0.0965 & -0.0353 \\
\hline $\mathrm{B}=\mathrm{C}^{\wedge} \mathrm{M}^{\wedge} \neg \mathrm{Y}$ & 0.0612 & 0.0961 & -0.0349 \\
\hline $\mathrm{K}=\mathrm{C}^{\wedge} \mathrm{M}^{\wedge} \mathrm{Y}$ & 0.0872 & 0.0514 & 0.0358 \\
\hline
\end{tabular}

These values were obtained by counting subpixels in a computer simulation of the in-phase and the counter-phase screen superpositions. The simulation has been done using three screens with identical circular dots having a constant radius of 0.35 screenperiod. 

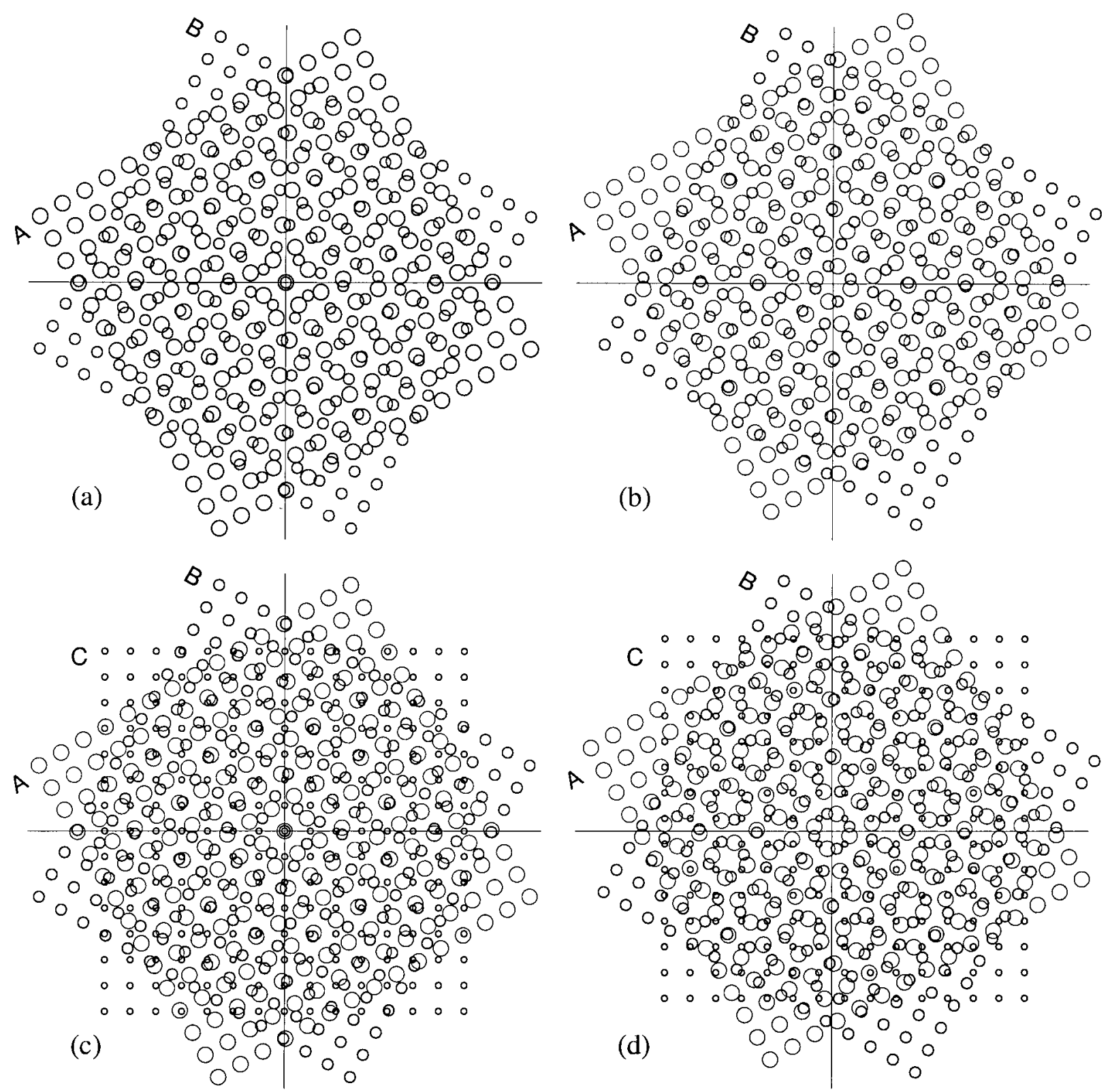

FIG. 2. A magnified view of the screen superpositions of Fig. 1, permitting to distinguish between the different layers and their precise dot locations.

A more formal demonstration of this phenomenon as well as its geometric interpretation can be found in Sec. 8.8 of Ref. 5.

In a similar way, as we can see from Figs. 3(c),(d) and Table II, in the in-phase superposition there is slightly less uncovered white space than in the counter-phase superposition. Here, again, we see that $P(\mathrm{~W})=P\left(\neg \mathrm{C}^{\wedge} \neg \mathrm{M}^{\wedge} \neg \mathrm{Y}\right)$ does vary with the registration shifts of the individual dot-screens. Although there may exist a particular combination in which $P\left(\neg \mathrm{C}^{\wedge} \neg \mathrm{M}^{\wedge} \neg \mathrm{Y}\right)=P(\neg \mathrm{C}) P(\neg \mathrm{M}) P(\neg \mathrm{Y})$, this equality is not true in all possible shifts, and in general we have

$$
P\left(\neg \mathrm{C}^{\wedge} \neg \mathrm{M}^{\wedge} \neg \mathrm{Y}\right) \neq P(\neg \mathrm{C}) P(\neg \mathrm{M}) P(\neg \mathrm{Y}) \text {. }
$$

These results clearly show that, in the case of the classical 3 -screen superposition, the Demichel equations do not hold.
Therefore, using them in the Neugebauer equations for the prediction of the colour obtained in the 3 -screen combination inevitably leads to some colorimetric errors depending on the precise registration shift between the 3 screens. Numeric examples of the colour variations due to such registration shifts can be found in Refs. 8 and 9; although usually these colour deviations are not excessively large, in some cases they may exceed $\Delta E_{L a b}$ of 10 , which is not a negligible value.

It is interesting to note, however, that such errors do not occur in the 2-screen counterpart of this classical 3-screen superposition. As we have seen above, in a 2-screen superposition with identical frequencies and an angle difference of $30^{\circ}$ (or $60^{\circ}$ ), no substantial microstructure changes occur due to layer shifts. This can be also verified in Table III: as we can easily see, all the ink combinations in this case 
TABLE III. The proportional areas of each of the $2^{2}=$ 4 possible combinations of ink superposition in the 2-screen ( $C$ and $M)$ counterpart of the classical 3-screen superposition, which is a nonsingular case, in in-phase and in counter-phase superpositions.

\begin{tabular}{lccr}
\hline Ink overlap & $\begin{array}{c}\text { Proportional } \\
\text { area in } \\
\text { in-phase } \\
\text { superposition }\end{array}$ & $\begin{array}{c}\text { Proportional } \\
\text { area in } \\
\text { counter-phase } \\
\text { superposition }\end{array}$ & Difference \\
\hline $\mathrm{W}=\neg \mathrm{C}^{\wedge} \neg \mathrm{M}$ & 0.3790 & 0.3789 & 0.0001 \\
$\mathrm{C}=\mathrm{C}^{\wedge} \neg \mathrm{M}$ & 0.2366 & 0.2375 & -0.0009 \\
$\mathrm{M}=\neg \mathrm{C}^{\wedge} \mathrm{M}$ & 0.2360 & 0.2361 & -0.0001 \\
$\mathrm{~B}=\mathrm{C}^{\wedge} \mathrm{M}$ & 0.1484 & 0.1475 & 0.0009 \\
\hline
\end{tabular}

These values were obtained by counting subpixels in a computer simulation of the in-phase and the counter-phase screen superpositions. The differences (see last column) are basically due to noise in the simulation process. The simulation has been done using two screens with identical circular dots having a constant radius of 0.35 screen-period.

remain insensitive to layer shifts. The particular case of white coverage is also illustrated in Figs. 3(a),(b); it is instructive to compare these figures with their 3 -screen counterparts, Figs. 3(c),(d).

This means that in 2-screen superpositions with identical frequencies and an angle difference of $30^{\circ}$ (or $60^{\circ}$ ) the two superposed screens are independent, and the Demichel equations do hold. However, as already shown in Ref. 2, this result is not true for all angle differences; and moreover, it turns out that this result depends also on the frequencies of the two superposed screens. How can we explain all these facts?

Although this behavior may seem surprising at first sight, in fact there is nothing mysterious about it. The answer is given by the following result (Ref. 5, Chapter 8, p. 224).

Proposition 1. A nontrivial shift of individual layers in the superposition causes a substantial change in the microstructure of the superposition if and only if their frequency vectors $\mathbf{f}_{i}$ are linearly dependent over $\mathbb{Z}$ (the set of all integer numbers), i.e., if and only if there exist integer numbers $k_{i}$ not all of them 0 such that $\sum k_{i} \mathbf{f}_{i}=\mathbf{0}$. But this precisely means that the superposition is singular.**

A short explanation of this proposition is provided in Appendix B. This proposition means, therefore, that the Demichel equations fail in all cases where the screen superposition is singular, and hold in all the nonsingular screen superpositions.

** A superposition is said to be singular if at least one integer linear combination of the frequency vectors of the original layers is exactly zero. This means that a superposition is singular, if it generates a moiré effect whose period is infinitely large, and hence invisible. However, singular states are very unstable moiré-free states, since any slight deviation in the angle or in the frequency of any of the superposed layers may cause the moiré to come back from infinity and to have a clearly visible period. The classical 3-screen superposition with angle differences of $30^{\circ}$ (or $60^{\circ}$ ) is, indeed, an example of a singular case, but its 2 -screen counterpart is not singular. For a more detailed discussion on singular and nonsingular superpositions, their image-domain and frequency-domain properties, and several examples and figures see Ref. 10, Sec. 4 or Ref. 5, Sec. 2.9.
Let us illustrate this result by means of a few additional examples:

1. The most trivial case of a singular screen superposition occurs when two (or more) screens with identical frequencies are superposed at the same angle. In this trivial case, the dot overlap of the screens may be anywhere between $0-100 \%$, depending on the registration shifts, and the Demichel equations obviously fail.

2. The 2-screen superposition of two identical dot-screens with an angle difference of $\arctan \left(\frac{3}{4}\right) \approx 36.87^{\circ}$ is singular, and, therefore, layer shifts may cause substantial changes in its microstructure [see Figs. 4(a),(b)]. And indeed, as already shown in Ref. 2, the Demichel equations in this case are not valid; this is clearly illustrated in Figs. 5(a),(b) and in Table IV.

3. Any stable moiré-free 2 -screen superposition, ${ }^{10}$ like the superposition of two identical screens with angle difference of $25^{\circ}, 30^{\circ}$, or $60^{\circ}$, is nonsingular; therefore, its microstructure consists of a homogeneous blend of rosettes of all types, and it is not substantially influenced by layer shifts [see Figs. 1(a),(b)]. And indeed, as already shown in Ref. 2, the Demichel equations in this case are valid.

4. Any stable moiré-free 3 -screen superposition ${ }^{10}$ is nonsingular; therefore, its microstructure consists of a homogeneous blend of rosettes of all types, and it is not substantially influenced by layer shifts. Once again, the Demichel equations in this case are valid.

5. The classical 4-screen superposition that is most often used in colour printing (i.e., angle differences of $30^{\circ}$ (or $60^{\circ}$ ) for the $\mathrm{C}, \mathrm{M}, \mathrm{K}$ screens and an angle difference of $15^{\circ}$ for the $\mathrm{Y}$ screen) is singular, because adding a fourth layer on top of a singular superposition does not affect the singularity. The Demichel equations in this case remain, therefore, nonvalid.

Examples (3) and (4) illustrate a particularly interesting consequence of our result. In cases of stable moiré-free superposition, ${ }^{10}$ where no moiré becomes visible even when a small deviation occurs in the angle or in the frequency of any of the superposed layers, the Demichel equations are valid. Note that such superpositions also offer a good colorimetric stability under registration shifts. Examples of such 3-screen superpositions are given in Fig. 19 of Ref. 10 and in Ref. 9. This is in contrast with unstable moiré-free superpositions, like the conventional 3- or 4-screen superpositions, which are by definition singular cases, and hence do not satisfy the Demichel equations.

Note, however, that although the above result tells us in which cases such deviations occur, it does not specify the degree of the discrepancy. In general, it turns out that the larger the number of superposed layers and the higher the order of the singular state (i.e., the larger the integers $k_{i}$ for which $\left.\sum k_{i} \mathbf{f}_{i}=\mathbf{0}\right)$ the less visible are its microstructure changes due to layer shifts in the superposition. And indeed, in such singular cases the colour deviations may become almost unperceptible-although they still do exist. 

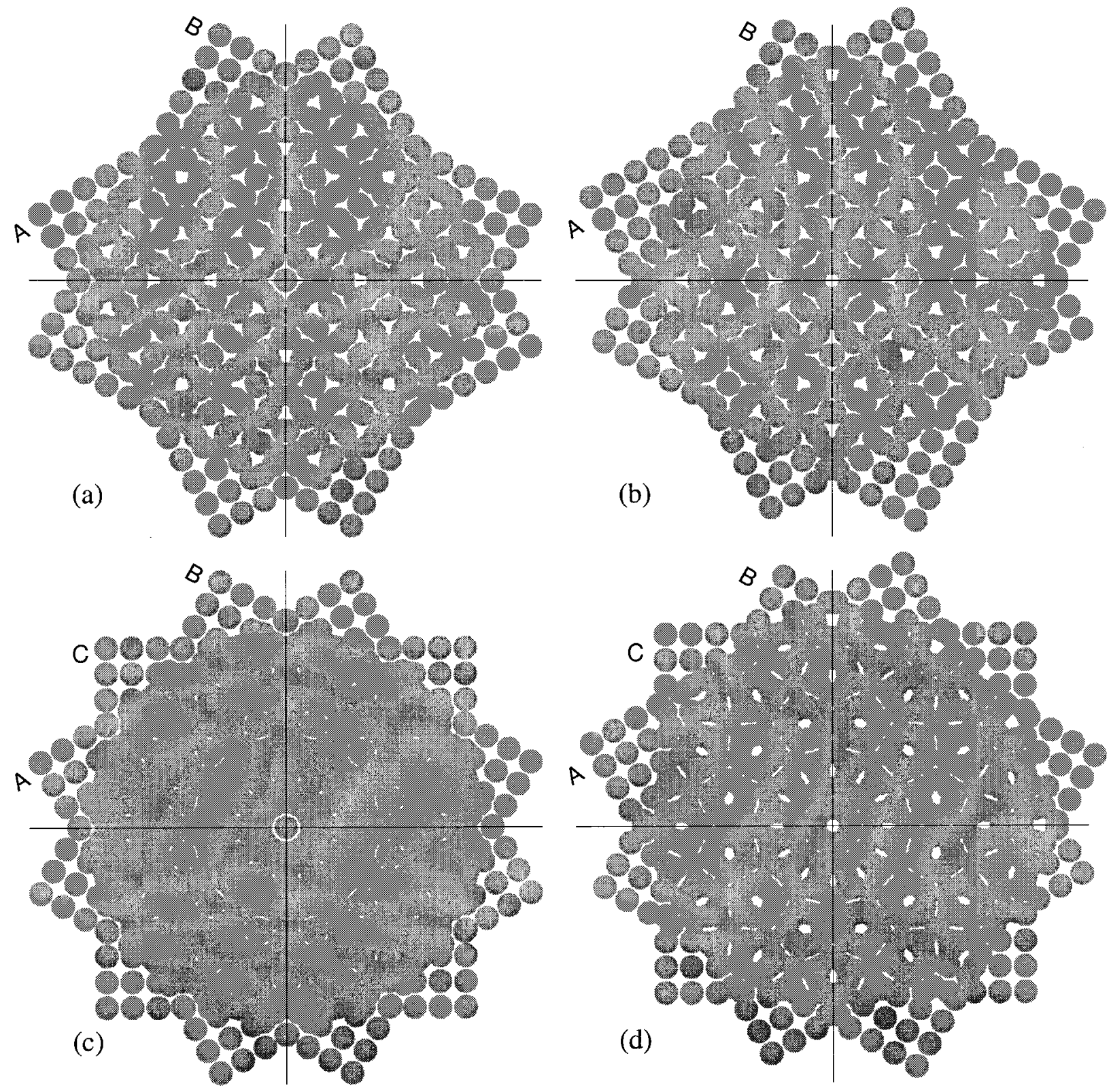

FIG. 3. (a) In-phase and (b) counter-phase superposition of two identical dot-screens with an angle difference of $30^{\circ}$. (c) In-phase and (d) counter-phase superposition of three identical dot-screens with angle differences of $30^{\circ}$. Note that in (c) there is slightly less uncovered white space than in (d), while between (a) and (b) no difference can be distinguished in the white coverage rate.

It is interesting to note that the Demichel equations are also valid in superpositions of random screens, where the statistical independence between the different screens is clearly guaranteed. Such random screens are currently gaining interest in the field of colour printing, and different variants have been recently developed..$^{11-13}$

\section{CONCLUSION}

Since the Demichel equations significantly simplify one's task when using the Neugebauer approach, it could be very helpful to have a simple criterion that determines for which screen superposition the Demichel equations can be used. A partial answer to this question has been recently given by Rogers, ${ }^{2}$ who showed that in the superposition of two regular dot-screens having the same frequency the validity of the Demichel equations depends on the angle between the two superposed screens.

In the present contribution, we extend this result to the most general case of $n$-screen superpositions with any angle and frequency combinations. We explain that the Demichel equations are valid only for screen superpositions which are nonsingular, while for singular superpositions, including the conventional 3- or 4-screen superpositions that are traditionally used in colour printing, the Demichel equations are not valid. Although the colour deviations that result from 


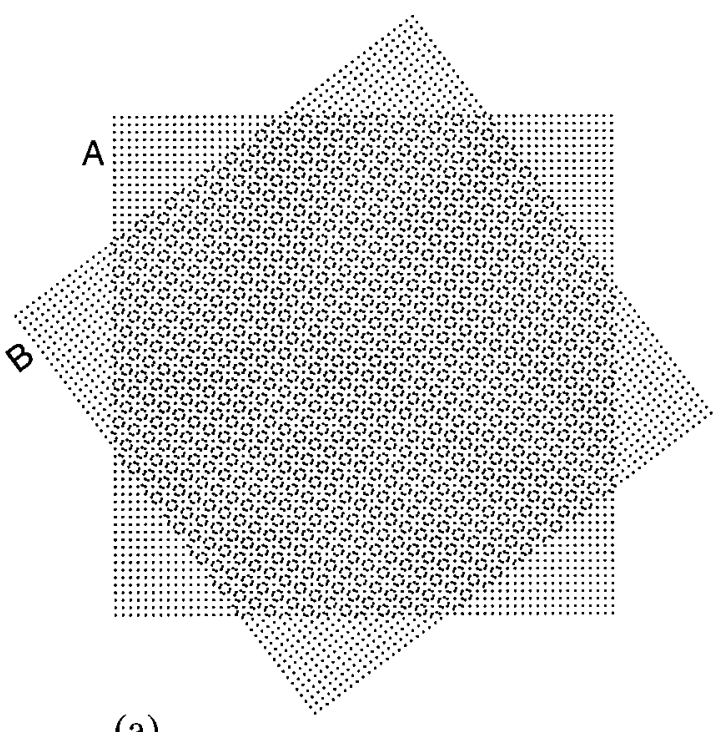

(a)

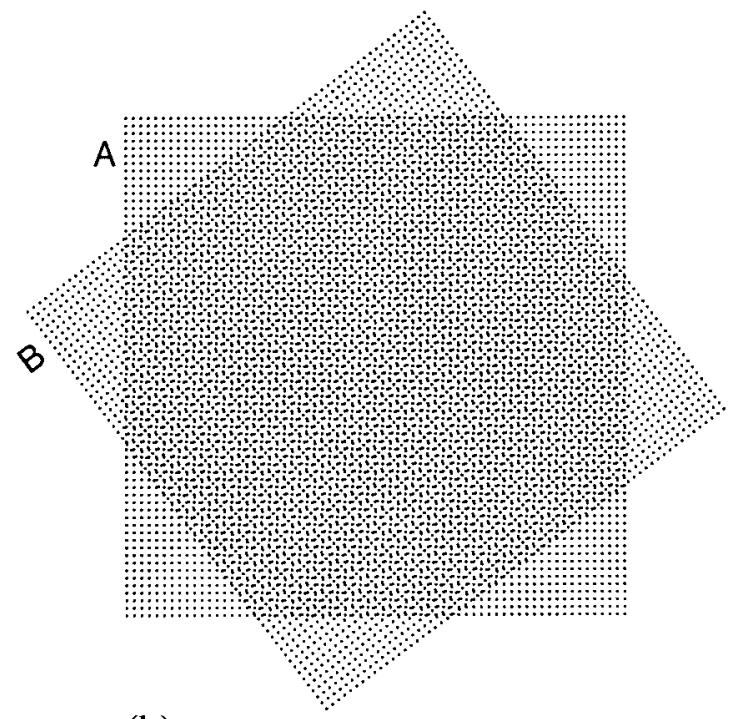

(b)

FIG. 4. The singular superposition of two identical dot-screens with angle difference of $\arctan \left(\frac{3}{4}\right) \approx 36.87^{\circ}$. (a): in-phase superposition; (b): counter-phase superposition.

using the Demichel equations in such singular cases are not excessively large, they still may be nonnegligible, and one should be aware of their existence when using the Neugebauer equations.

Finally, it should be mentioned that, although we used in this article 2-screen and 3-screen superpositions as our main examples, the Neugebauer and the Demichel equations as well as all our results are completely general, for any $n$-screen superposition.

\section{APPENDIX A: INDEPENDENCE AND RANDOMNESS}

The basic condition required for the Demichel equations to hold is, of course, that the superposed screens be independent of each other, since only in this case we have

$$
\begin{gathered}
P\left(\mathrm{C}^{\wedge} \mathrm{M}\right)=P(\mathrm{C}) P(\mathrm{M}) \\
P\left(\mathrm{C}^{\wedge} \mathrm{M}^{\wedge} \mathrm{Y}\right)=P(\mathrm{C}) P(\mathrm{M}) P(\mathrm{Y}),
\end{gathered}
$$

etc. However, it is a very common mistake to say that the requirement for the Demichel equations to hold is that "the dot pattern in the screen superposition be distributed as though it were a random pattern," or that "the dots of each screen be randomly positioned with respect to the dots of the other screens." The mistake in such statements is that they confuse the two different notions of "being independent" and "being random." But these two notions are not equivalent: while randomness of the different layers implies their independence, their independence does not necessarily imply randomness. Thus, it is true that if the dots in each of the C, M, Y layers were randomly distributed, then in the superposition the 3 layers would be independent of each other; but it is important to understand that even when each of the layers is perfectly ordered and deterministic, as is the case in regular dot-screens, they still can be independent of each other. Proposition 1 precisely states in which cases superposed regular dot-screens are independent, and in which cases they are not.

Note that, in fact, superposed layers can be independent of each other even if they are not screened at all, but rather partially covered by a single area of continuously deposited ink, as shown in Fig. 6(a). In all cases, screened or not, the probabilities $P(\mathrm{C}), P(\mathrm{M})$, and $P\left(\mathrm{C}^{\wedge} \mathrm{M}\right)$ should be understood as "the probabilities that a randomly chosen point (or pixel) within the given layer superposition falls in the range covered by the inks C, M, or both." But it is completely irrelevant whether the layers in question and their superposition are themselves random or not.

Note, however, that although in cases like Fig. 6(a) the Demichel equations do hold, they are, in fact, useless, since the different colour zones here are too large to be blended

TABLE IV. The proportional areas of each of the $2^{2}$ $=4$ possible combinations of ink superposition in the singular 2-screen superposition ( $C$ and $M)$ with angle difference of $\arctan \left(\frac{3}{4}\right) \approx 36.87^{\circ}$.

\begin{tabular}{lccr} 
Ink overlap & $\begin{array}{c}\text { Proportional } \\
\text { area in } \\
\text { in-phase } \\
\text { superposition }\end{array}$ & $\begin{array}{c}\text { Proportional } \\
\text { area in } \\
\text { counter-phase } \\
\text { superposition }\end{array}$ & Difference \\
\hline $\mathrm{W}=\neg \mathrm{C}^{\wedge} \neg \mathrm{M}$ & 0.3918 & 0.3693 & 0.0225 \\
$\mathrm{C}=\mathrm{C}^{\wedge} \neg \mathrm{M}$ & 0.2251 & 0.2476 & -0.0225 \\
$\mathrm{M}=\neg \mathrm{C}^{\wedge} \mathrm{M}$ & 0.2232 & 0.2457 & -0.0225 \\
$\mathrm{~B}=\mathrm{C}^{\wedge} \mathrm{M}$ & 0.1599 & 0.1374 & 0.0225 \\
\hline
\end{tabular}

These values were obtained by counting subpixels in a computer simulation of the in-phase and the counter-phase screen superpositions. The simulation has been done using two screens with identical circular dots having a constant radius of 0.35 screen-period. 

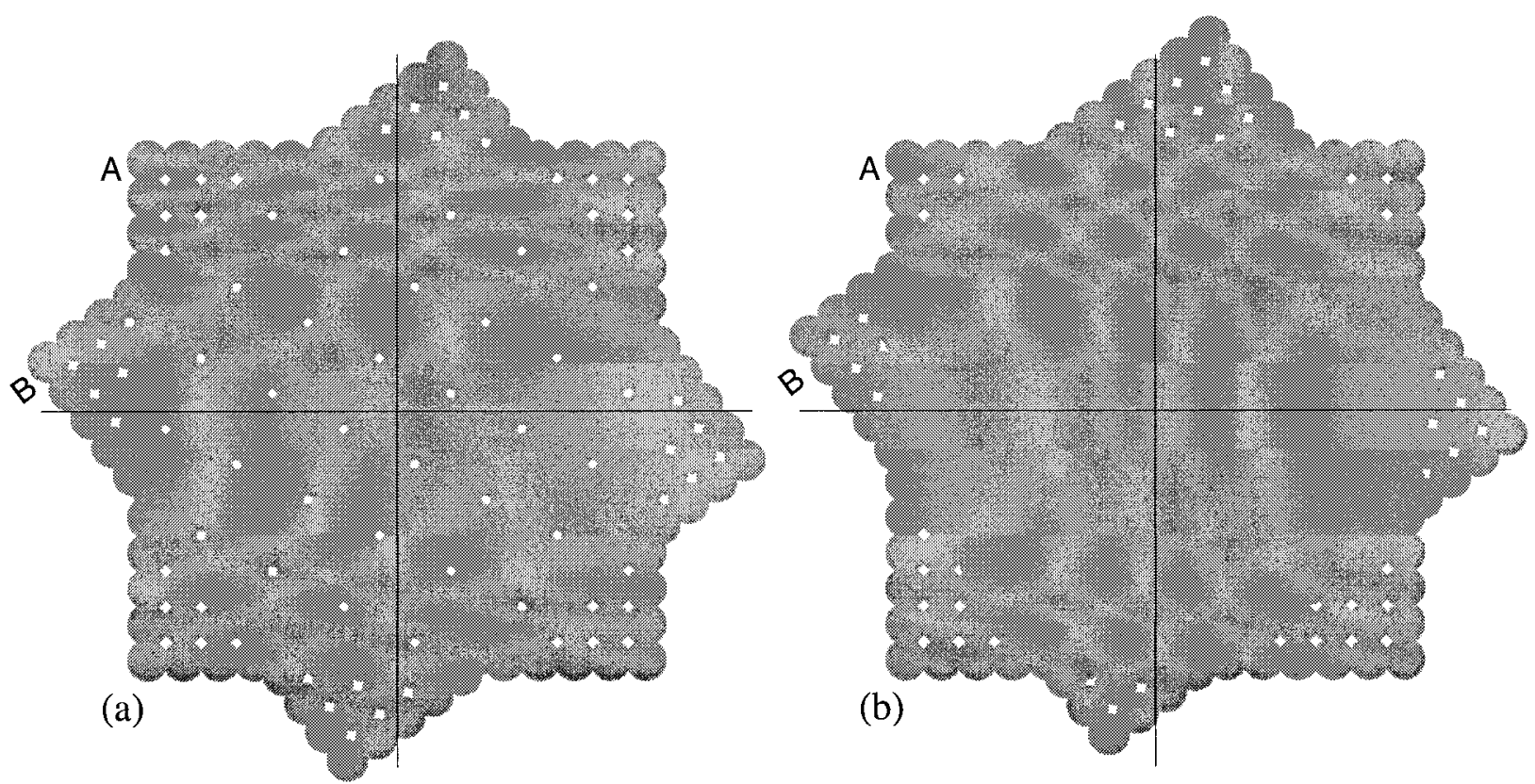

FIG. 5. (a) In-phase and (b) counter-phase superposition of two identical dot-screens with an angle difference of arctan $\left(\frac{3}{4}\right) \approx$ $36.87^{\circ}$ : a magnified view of Fig. 4 with larger dots. Note that in (a) there is slightly more uncovered white space than in (b).

together by the eye as an additive colour composition, so that the Neugebauer equations can no longer be used.

\section{APPENDIX B: EXPLANATION OF PROPOSITION 1}

As we have seen earlier in this article, when periodic layers (such as line grids, dot-screens, etc.) are superposed, new

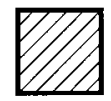

Area covered by solid (non screened) cyan ink

Area covered by solid (non screened) magenta ink

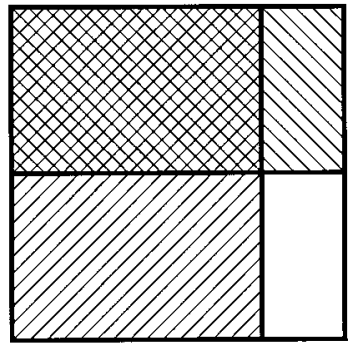

(a)

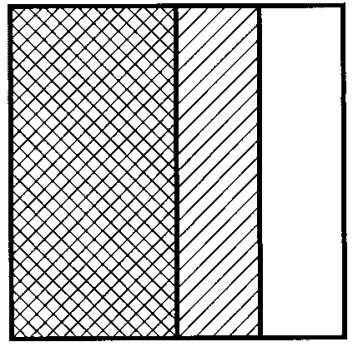

(b)
FIG. 6. (a) The superposition of a solid (nonscreened) cyan ink layer and a solid magenta ink layer that are independent of each other and, hence, satisfy $P\left(\mathrm{C}^{\wedge} \mathrm{M}\right)=P(\mathrm{C}) P(\mathrm{M})$. (b) An example where the superposed layers are not independent and, hence, $P\left(\mathrm{C}^{\wedge} \mathrm{M}\right) \neq P(\mathrm{C}) P(\mathrm{M})$. Note that $P(\mathrm{C})$ and $P(\mathrm{M})$ are identical in cases (a) and (b), but $P\left(\mathrm{C}^{\wedge} \mathrm{M}\right)$ is different in each case. structures of two distinct levels may appear in the superposition, which do not exist in any of the original layers: the macrostructures and the microstructures.

The macrostructures, usually known as the moiré patterns, are, of course, the most prominent; being much coarser than the detail of the original layers, they are clearly visible even when observed from a distance. A few examples of such moirés that occur in the superposition of two or three regular dot-screens are shown in Figs. 7(a)-(d). The right-hand side of each figure shows the interpretations of these moirés in the frequency domain (= the Fourier spectral domain). Each of these moirés corresponds to a certain vectorial sum or difference of the frequency vectors $\mathbf{f}_{i}$ of the original superposed periodic screens. Note that each of the screens contributes to the spectrum two orthogonal frequency vectors that correspond to its frequencies in its two main directions, and each 2D moiré is also represented by two orthogonal frequency vectors (vectorial sums). For example, the moiré effect which is visible in Fig. 7(a) corresponds to the frequency vector difference $\mathbf{f}_{1}-\mathbf{f}_{3}$ and its orthogonal counterpart $\mathbf{f}_{2}-\mathbf{f}_{4}$, while the moiré effect in Fig. 7 (c) corresponds to the frequency vector sum $\mathbf{f}_{1}+2 \mathbf{f}_{2}-$ $2 \mathbf{f}_{3}-\mathbf{f}_{4}$ and its orthogonal counterpart $-2 \mathbf{f}_{1}+\mathbf{f}_{2}+\mathbf{f}_{3}-$ $2 \mathbf{f}_{4}$. It is important to note, however, that the moiré effect is visible only when the corresponding vectorial frequency sum, $\sum k_{i} \mathbf{f}_{i}$, falls in the spectrum within the visibility circle. The visibility circle is a circular step function around the spectrum origin whose radius corresponds to the cutoff frequency of the eye at the corresponding viewing conditions (distance, illumination, etc.), i.e., to the threshold

\footnotetext{
* For a short introduction to the frequency analysis of grating or screen superpositions, see Ref. 10 or Chapter 2 in Ref. 5.
} 


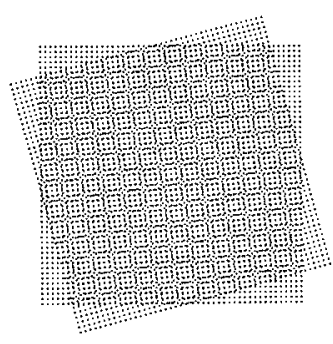

(a): $\alpha=15^{\circ}, T_{1}=T_{2}=T_{3}=T_{4}$
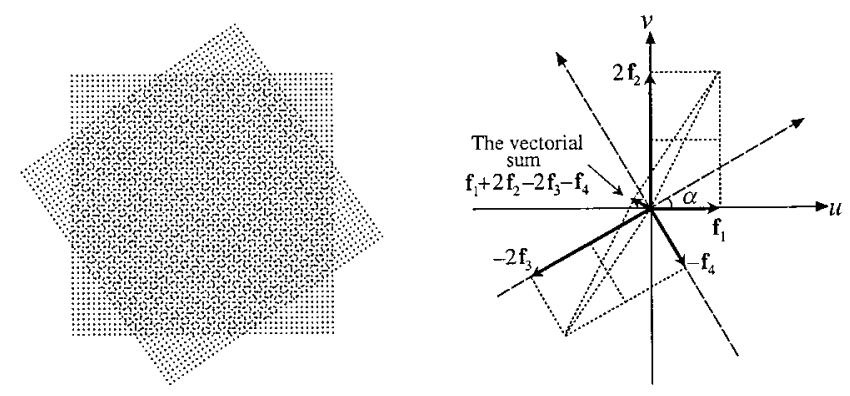

(c): $\alpha=34.5^{\circ}, T_{1}=T_{2}=T_{3}=T_{4}$
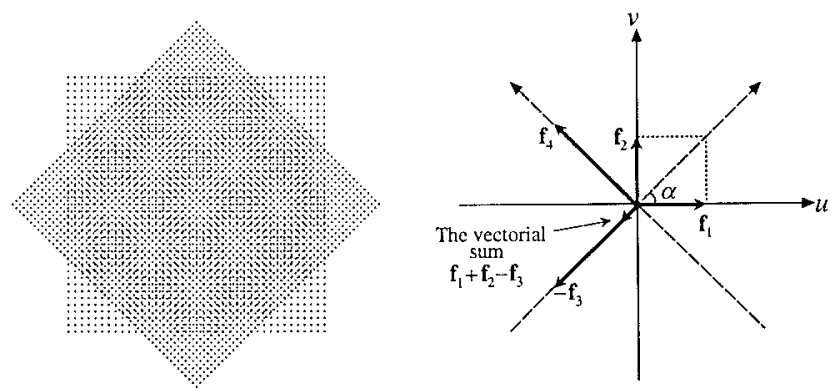

(b): $\alpha=45^{\circ}, T_{1}=T_{2}=1.3 T_{3}=1.3 T_{4}$
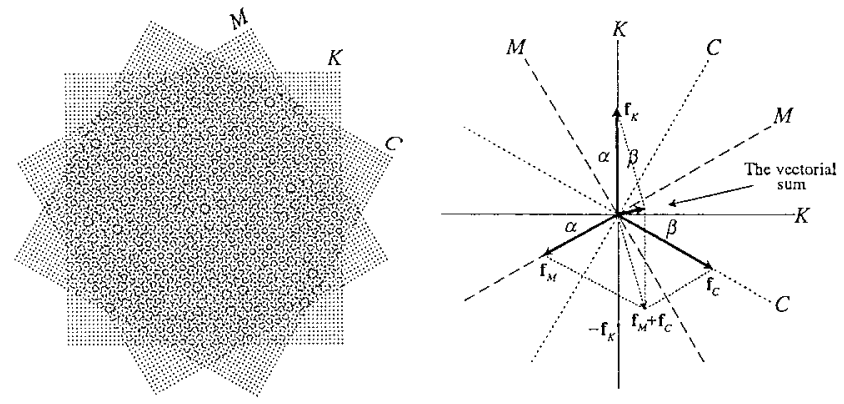

(d) $: \alpha=30^{\circ}, \beta=30^{\circ}, T_{1}=T_{2}=0.97 T_{3}=0.97 T_{4}=1.02 T_{5}=1.02 T_{6}$

FIG. 7. (a)-(c) Three examples of different moirés between two dot-screens (left), and their spectral interpretations (right). (d) (left) A three dot-screen moiré and (right) its spectral interpretation. For the sake of clarity, only one of the two perpendicular frequency vectors of the moiré is shown in each case. Dashed axes belong to the rotated layers. $T_{1}, T_{2} ; T_{3}, T_{4}$ and $T_{5}, T_{6}$ are the periods of the first, the second, and the third screens along their two main axes.

frequency beyond which fine detail is no longer detected by the eye. ${ }^{\S}$ Thus, when the vectorial sum of the moiré falls beyond the visibility circle, the period of the moiré is too small to be detected by the eye, and the moiré is no longer visible. However, there exists also another case in which the moiré effect is not visible: this occurs when the vectorial frequency sum of the moiré is exactly zero, and it falls on the spectrum origin. In this case, the period of the moiré effect is infinitely large, so that it is no longer visible in any finite-sized part of the superposition. The moiré in this case is said to be singular ${ }^{10}$; we also say in such cases that the superposition is singular.

In contrast to macrostructures, the microstructures generated in the superposition are almost as small as the periods of the original screens (typically, just 2-5 times larger), and, therefore, they are visible only when examining the superposition from a close distance or through a magnifying glass. These tiny structures are also called rosettes due to the various flower-like shapes they often form in the superposition of dot-screens (Ref. 6, p. 339).

It is important to note that macrostructures and microstructures coexist in the same superpositions; in fact, macrostructures, whenever they exist, are made up from repetitive alternations in the microstructures. It is particularly interesting to note that the microstructure alternations that

\footnotetext{
$\S$ Obviously, the visibility circle is just a schematic approximation; in reality, this is a bidimensional bell-shaped low-pass filter whose form is not really circular but rather anisotropic, since it appears that the eye is less sensitive to small details in diagonal directions such as $45^{\circ}$.
}

make up a macro-moiré are, to a very close approximation, nothing else but the microstructure forms that are obtained at the singular state of that macro-moiré in all possible phase shifts of the superposed layers (Ref. 5, Sec. 8.4). The two extreme "in-phase" and "counter-phase" microstructures (e.g., the "dot-centered" and the "clear-centered" rosettes in the case of the classical 3-screen superposition) generate the two extreme intensity levels of the visible macro-moiré (its brightest and darkest areas), and the intermediate forms between them generate all the in-between intensity levels of the macro-moiré. This result can be observed, for example, by comparing the local microstructures in the dark and in the clear areas of Fig. 7(d) with the microstructures in Figs. 1(c) and 1(d), or by comparing the local microstructures of Fig. 7(c) with the microstructures in Figs. 4(a) and 4(b).

Another interesting microstructure interpretation of a macro phenomenon in the screen superposition concerns singular moirés. When a moiré effect is singular, its period is infinitely large; this means that in any finite-sized part of the superposition we can only see one of its different microstructures, the others being located infinitely far.

With this background in mind, we are now ready to explain Proposition 1.

Suppose, first, that in the given screen superposition we have $\sum k_{i} \mathbf{f}_{i} \approx \mathbf{0}$, so that in the spectrum of the superposition the vector sum $\sum k_{i} \mathbf{f}_{i}$ is located within the visibility circle. This vector sum represents, therefore, a moiré effect that is visible in the screen superposition. Microscopically speak- 
ing, this moiré is composed of alternate areas in the superposition, which contain rosettes of the in-phase superposition, the counter-phase superposition, and all their intermediate layer shifts. Now, if we slowly shift the superposed screens on top of each other, without changing their periods and their orientations, the moire effect in the superposition is shifted across the superposition (see Result 2 in Ref. 14). This means that while the superposed layers are being shifted, we see within a small window around the origin, as the moiré periods pass through it, a succession of rosette forms from all the different shift positions of the screens. Now, suppose that we slightly modify the vectors $\mathbf{f}_{i}$ (i.e., that we slightly stretch and rotate the superposed layers) so that $\sum k_{i} \mathbf{f}_{i}$ tends to $\mathbf{0}$. The period of the moiré gradually gets larger and larger, and the alternate rosette zones appear farther apart; but still, as the layers are shifted on top of each other, we see in our small window a succession of rosette zones of all different forms. At the moment when $\sum k_{i} \mathbf{f}_{i}=\mathbf{0}$, the frequency of our moiré is zero and its period is infinite, meaning that the moire is singular. Since the singular moire is no longer visible, only one type of rosette is visible in our window, the other rosette types being located now infinitely far away, within the infinite moiré period. When the layers in this singular state are shifted on top of each other, we see at each moment a different type of rosette throughout the window as the infinite moiré period moves across, but this time the rosette shapes at each shift position will be uniform throughout any finite-sized window, i.e. throughout the whole superposition. This means, indeed, that when the screen superposition is singular, a nontrivial shift of individual layers in the superposition causes a substantial change in the microstructure throughout the superposition. While at some shift positions we see only "in-phase" rosettes, in other shift positions we see only "counter-phase" rosettes, or intermediate rosette types between them. This is illustrated in Figs. 1 (c),(d) for the conventional singular 3-screen superposition, and in Figs. 4(a),(b) for a typical singular 2-screen superposition. Now, on the contrary, when no moiré in the superposition is singular, the periods of the moirés are not infinite but either visible (if the corresponding vector sum $\sum k_{i} \mathbf{f}_{i}$ is inside the visibility circle), or too small for being visible (if the vector sum is outside the visibility circle). In both cases, layer shifts simply shift the locations of the different rosette types in the superposition following the motion of the moiré periods, but no uniform global rosetteshape change occurs in the superposition.

This explains, indeed, Proposition 1: A nontrivial shift of individual layers in the superposition causes a substantial change in the microstructure of the superposition if and only if the superposition is singular.

1. Rhodes W. Fifty years of the Neugebauer equations. SPIE Vol. 1184: Neugebauer memorial seminar on colour reproduction; 1989. p 7-18.

2. Rogers GL. Neugebauer revisited: random dots in halftone screening. Col Res Appl 1998;23:104-113.

3. Hunt RWG. The reproduction of colour in photography, printing and television. 4th Ed. London: Fountain; 1987. p 20-24.

4. Demichel E. Le procédé. 1924;26:17-21, 26-27.

5. Amidror I. The theory of the moiré phenomenon. Dordrecht: Kluwer; 2000 .

6. Yule JAC. Principles of color reproduction. New York: Wiley; 1967. p 328-345.

7. Delabastita PA. Screening techniques, moiré in four color printing. Vancouver: Proc TAGA Conf; 1992. p 44-65.

8. Daels K, Delabastita P. Color balance in conventional halftoning. Baltimore: Proc TAGA Conf; 1994. p 1-18.

9. Schoppmeyer J. Screen systems for multicolor printing. U.S. Patent No. 4,537,470, 1985 .

10. Amidror I, Hersch RD, Ostromoukhov V. Spectral analysis and minimization of moiré patterns in colour separation. J Elec Imag 1994;3: 295-317.

11. Widmer E, Schläpfer K, Humbel V, Persiev S. The benefits of frequency modulation screening. Vancouver: Proc TAGA Conf; 1992. p $28-43$.

12. Schläpfer K. Are fine screens an alternative to frequency modulation screening. Baltimore: Proc TAGA Conf; 1994. p 34-41.

13. Ostromoukhov V. Pseudo-random halftone screening for color and black\&white printing. Yokohama: Proc 9-th Int Cong Non-impact Print Tech; 1993. p 579-582.

14. Amidror I, Hersch RD. Fourier-based analysis of phase shifts in the superposition of periodic layers and their moiré effects. J Opt Soc Am A 1996;13:974-987. 